\title{
Characteristics of connective tissue diseases associated interstitial lung diseases - a single centre study in Sri Lanka
}

\author{
Prasanjanie Jayasinghe ${ }^{1}$, Nuwan Wickramasinghe ${ }^{2}$, Prathapan Krishnakumar ${ }^{1}$, Aflah Sadikeen ${ }^{1}$, \\ Amitha Fernando ${ }^{1}$
}

(Index words: connective tissue diseases, Interstitial lung disease, pattern of HRCT chest

\begin{abstract}
Introduction Interstitial lung diseases are the major cause of mortality in connective tissue diseases.

Objectives Our aim is to describe patients with interstitial lung disease associated with connective tissue diseases (CTD-ILD) in the Chest Clinic, Colombo 08.

Methods We conducted a descriptive cross-sectional study at the Central Chest Clinic, Colombo, Sri Lanka and data of all patients attending the Clinic were analysed.

Results The sample consisted of 83 consecutive patients diagnosed with CTD-ILD. The age ranged from 24 to 72 years with mean (SD) age of 55.6 (10.6) years. The majority was female patients $(n=70,88.6 \%)$. The majority $53.0 \%(n=44)$ had RA and SSc was the second commonest CTD-ILD ( $n=22,26.5 \%)$. NSIP 51(61.9\%) was the most frequently observed HRCT pattern in our cohort whereas UIP was the second commonest.

RA-ILD subgroup demonstrated female $(n=37,86.0 \%)$ preponderance. NSIP ( $n=24,55.8 \%)$ was the commonly observed HRCT pattern followed by UIP ( $n=15,34.9 \%)$ pattern. Among RA-ILD patients, 28 (65.1\%) had positive Rheumatoid factor. Majority of $(n=20,90.9 \%)$ SSc-ILD were females. Sixteen (72.7\%) of them had NSIP pattern in HRCT analysis followed by UIP ( $n=6,27.3 \%)$.

Conclusion RA-ILD was the most frequent type of CTDILD followed by SSc. More importantly; the present study revealed the predominant NSIP pattern and clear female preponderance in RA-ILD compared to global data. We recommend prospective multicenter studies to be carried out and prospective disease registries to be established to explore the epidemiological, clinical, radiological and prognostic characteristics of CTD-ILD in Sri Lanka.
\end{abstract}

Ceylon Medical Journal 2021; 66: 38-43

DOI: http://doi.org/10.4038/cmj.v66i1.9356

\section{Introduction}

The connective tissue diseases (CTDs) are multisystemic rheumatological diseases characterized by autoimmune mediated disease process causing multiorgan dysfunction. Among the multiple causes leading to mortality in CTD patients, interstitial lung diseases (ILD) are identified as the main cause [1].

CTDs associated ILDs (CTD-ILD) have been described in rheumatoid arthritis (RA), systemic sclerosis (SSc), Inflammatory myositis (IM), primary Sjögren's syndrome (pSS) and mixed connective tissue disease (MCTD) [2]. In addition, there is another entity called interstitial pneumonitis with autoimmune features (IPAF) [3], which is described recently. Nonspecific pneumonia (NSIP) and usual interstitial pneumonia (UIP), organizing pneumonia (OP), diffuse alveolar damage (DAD) and lymphocytic interstitial pneumonia (LIP) are the main histopathological subtypes identified in CTD-ILD [4].

Identification of the type of ILD is necessary to decide on definitive treatment and more importantly to prognosticate the disease. Interestingly, most of the time High Resolution CT scan (HRCT) patterns reflect the underlying histopathological subtypes [4]. Studies have shown that in UIP, HRCT diagnosis has excellent concordance with the histological diagnosis [5]. Lung biopsy is recommended only in atypical cases [6]. Thus, identification of the pattern of HRCT is mandatory to decide definitive treatment and assess the prognosis.

It is Important to note that limited studies have been conducted regarding histological and radiological pattern of CTD-ILD in Sri Lanka [7]. Available data also being obtained from the studies conducted in the other countries [8]. In practice, these findings are extrapolated to various clinical settings across the globe including Sri Lanka. However, it is important to generate local evidence base in the Sri Lankan population in order to identify any possible differences compared to the western population.

${ }^{1}$ Central Chest Clinic and National Hospital of Sri Lanka, ${ }^{2}$ Faculty of Medicine and Allied Sciences, Rajarata University of Sri Lanka.

Correspondence: PJ, e-mail: <p_jayasinghe@yahoo.com>. Received 16 January 2021 and revised version 17 March 2021 accepted 20 March 2021.

This is an open-access article distributed under the terms of the Creative Commons Attribution License, which permits unrestricted use, distribution, and reproduction in any medium, provided the original author and source are credited. 
Many sociodemographic and immunological factors are identified as risk factors of developing ILD [8]. As an example, male gender, severity of the RA, older age, presence of rheumatoid factor, anti CCP antibody and smoking carry risk for developing RA-ILD [9]. This further emphasizes the necessity of data from our population, which has many sociodemographic differences compared to European countries. Hence, it is important to identify which subtype of ILD is predominant in the Sri Lankan population. Accordingly, the available diagnostic and treatment facilities could be further developed to cater the needs of the local setting.

\section{Methods}

We conducted a descriptive cross-sectional study at Central Chest Clinic, Colombo, Sri Lanka, which is the largest chest clinic in the country. CTD-ILD patients, who met 2010American College of Rheumatology and European League Against Rheumatism (ACR/EULAR) classification criteria for RA, 2013 ACR/EULAR classification criteria for SSc, 2017 ACR/EULAR classification criteria for DM/ PM, 2016 ACR/EULAR classification criteria for pSS and 2015 ERS diagnostic criteria for IPAF were included in the study. Diagnosis of ILD was made on the basis of the HRCT findings.

Information on socio-demographic data, clinical features and autoantibodies were recorded by reviewing patient medical records. All HRCT findings, which were evaluated at the multidisciplinary team meeting by an experienced Radiologist and a Consultant Chest Physician were documented.

Data was collected after obtaining the ethical clearance from Medical Research Institute (MRI) in Sri Lanka, informed written consent of the patient and permission from the hospital director.

Data analysis was carried out using SPSS version 25.0. Descriptive statistics including mean [Standard Deviation (SD)], median [Inter-quartile range (IQR)] and frequency distributions were used to present study variables. Fisher's exact test was used to compare the differences of categorical variables between subgroups.

\section{Results}

\section{Sample characteristics}

The sample consisted of 83 consecutive patients diagnosed with CTD-ILD attending the Central Chest Clinic Colombo 08. The age ranged from 24 to 72 years and the mean (SD) age was 55.6 (10.6) years. Mean age of female and male were 55.0 and 58.7 respectively. The majority was female patients ( $\mathrm{n}=70,88.6 \%)$.

Time of the initial diagnosis was recorded in 55 subjects. CTD was diagnosed before ILD in 53 (96.4\%) of them, with a median (IQR) interval of 4 (2-8) years separating the two diagnoses. ILD was diagnosed first only in two (3.6\%) patients.

\section{Distribution of CTD-ILD}

The distribution of the CTD-ILD in the sample is given in Table 1. The majority 53.0\% $(n=44)$ had RA and SSc was the second commonest CTD-ILD ( $n=22,26.5 \%)$.

\section{HRCT patterns of ILD}

$\operatorname{NSIP}(n=51,61.9 \%)$ was the most frequently observed HRCT pattern among the CTD-ILD patients, whereas UIP was the second commonest pattern $(n=25,30.8 \%)$ Details of the HRCT patterns are given in Table 2.

Table 1. Distribution of CTD-ILD in the sample

\begin{tabular}{lcc}
\hline CTD-ILD & Number & Percentage \\
\hline Rheumatoid arthritis & 44 & $53.0 \%$ \\
Systemic sclerosis & 22 & $26.5 \%$ \\
$\begin{array}{l}\text { Mixed connective tissue } \\
\text { diseases Overlap syndrome }\end{array}$ & 3 & $3.6 \%$ \\
$\begin{array}{l}\text { Inflammatory myositis } \\
\begin{array}{l}\text { Systemic lupus } \\
\text { erythematosus (SLE) }\end{array}\end{array}$ & 4 & $4.8 \%$ \\
$\begin{array}{l}\text { Interstitial pneumonias with } \\
\text { autoimmune features (IPAF) }\end{array}$ & 3 & $2.4 \%$ \\
& & \\
\hline
\end{tabular}

Table 2. HRCT patterns of CTD-ILD

\begin{tabular}{lcc}
\hline HRCT Pattern & Number & Percentage \\
\hline NSIP & 51 & 61.9 \\
UIP & 25 & 30.8 \\
HP & 3 & 3.7 \\
OP & 1 & 1.2 \\
CPEF & 1 & 1.2 \\
\hline
\end{tabular}

\section{Clinical features}

Shortness of breath and cough were the commonest presenting symptoms found in 55 (66.3\%) and 49 (59.0\%) patients respectively. Fine crepitations on auscultation of lungs was noted among 43 (51.8\%) of the sample. One fifth of the sample had fatigue $(n=17,20.5 \%)$.

\section{Pulmonary function test interpretation}

Records on pulmonary function tests done at initial presentation were available in 55 patients. Restrictive pattern 47(85.4\%) (FVC and FEV1 being $<80 \%$ predicted with FEV1/FVC $>70 \%$ ) was observed in the majority. Six (10.9\%) of them had normal spirometry followed by obstructive $(n=1,1.8 \%)$ and mixed pattern $(n=1,1.8 \%)$. 
Characteristics of patients with common ILDs

Rheumatoid arthritis

RA-ILD subgroup demonstrated female ( $n=37,86.0 \%$ ) preponderance. NSIP ( $\mathrm{n}=24,55.8 \%$ ) was the commonly observed HRCT pattern followed by UIP ( $n=15,34.9 \%)$ pattern. In this sub-group, there were three patients with hypersensitive pneumonitis as well.

Shortness of breath $(n=28,65.1 \%)$ and cough $(n=20$, $46.5 \%$ ) were documented as the common presenting symptoms. Among RA-ILD patients, 28 (65.1\%) had positive rheumatoid factor.

Comparison of RA-ILD [RA (+)] group with other CTDILD [RA (-)] group

The mean (SD) age of the RA (-) and RA (+) groups were 52.3 (11.3) years and 58.7 (8.9) years respectively. There was a statistically significant difference in the mean age between the two groups $(\mathrm{t}(77)=-2.824, \mathrm{p}=0.006)$.

There were no statistically significant associations between RA and gender, HRCT patterns or symptomatology ( $p>0.05)$. However, there was a statistically significant association between RA and rheumatoid factor positivity (Chi (1) = $24.8675 \mathrm{p}<0.001)$.

Systemic sclerosis

Majority of ( $n=20,90.9 \%)$ SSc-ILD were females. Sixteen (72.7\%) of them had NSIP pattern in HRCT analysis followed by UIP ( $n=6,27.3 \%)$. Most of them had shortness of breath $(n=16,72.7 \%)$ and cough $(n=13,59.1 \%)$ on presentation. Majority $(\mathrm{n}=15,68.2 \%)$ had bilateral fine end-inspiratory crepitations on examination.

Comparison of SSc-ILD [SS (+)] group with other CTDILD [SS (-)] group

The mean (SD) age of the SS (-) and SS (+) groups were 57.8 (9.7) years and 49.9 (10.8) years respectively. There was a statistically significant difference in the mean age between the two groups $(\mathrm{t}(77)=3.128, \mathrm{p}=0.002)$.

There were no statistically significant associations between SSc and gender, HRCT patterns or symptomatology $(p>0.05)$. However, there were statistically significant associations between SSc and rheumatoid factor positivity [Chi $(1)=10.9691, \mathrm{p}=0.001]$ and SSc and ANA positivity [Chi $(1)=0.9568, \mathrm{p}=0.005]$.

Table 3. Comparison of RA-ILD [RA (+)] group with other CTD-ILD [RA (-)] group

\begin{tabular}{|c|c|c|c|}
\hline Characteristic & $R A(-) n(\%)$ & $R A(+) n(\%)$ & Statistical significance \\
\hline \multicolumn{4}{|l|}{ Gender } \\
\hline Male & $4(44.4)$ & $5(55.5)$ & Chi $(1)=0.754$ \\
\hline Female & $33(47.1)$ & $37(52.8)$ & $\mathrm{p}=0.460$ \\
\hline \multicolumn{4}{|l|}{ HRCT } \\
\hline NSIP & $27(57.9)$ & $24(47.0) 8$ & Chi $(1)=5.8903$ \\
\hline UIP & $10(4)$ & $15(6)$ & $\mathrm{p}=0.207$ \\
\hline HP & 0 & $3(100)$ & \\
\hline $\mathrm{OP}$ & $1(100)$ & 0 & \\
\hline CPEF & 0 & $1(100)$ & \\
\hline \multicolumn{4}{|c|}{ Shortness of breath } \\
\hline Absent & $12(42.8)$ & $16(57.1)$ & Chi $(1)=0.2895$ \\
\hline Present & $27(49.0)$ & $28(50.9)$ & $\mathrm{p}=0.647$ \\
\hline \multicolumn{4}{|l|}{ Cough } \\
\hline Absent & $20(58.8)$ & $14(41.1)$ & Chi $(1)=3.2388$ \\
\hline Present & $19(38.7)$ & $30(61.2)$ & $\mathrm{p}=0.080$ \\
\hline \multicolumn{4}{|l|}{ Fatigue } \\
\hline Absent & $30(45.4)$ & $36(54.5)$ & Chi $(1)=0.3942$ \\
\hline Present & $9(52.9)$ & $8(47.8)$ & $\mathrm{p}=0.599$ \\
\hline \multicolumn{4}{|c|}{ Bilateral fine end-inspiratory crepitations } \\
\hline Absent & $18(4)$ & $22(5)$ & Chi $(1)=0.1225$ \\
\hline Present & $21(48.8)$ & $22(51.1)$ & $\mathrm{p}=0.827$ \\
\hline \multicolumn{4}{|c|}{ Rheumatoid factor } \\
\hline Absent & $35(68.6)$ & $16(31.3)$ & Chi $(1)=24.8675$ \\
\hline Present & $4(12.5)$ & $28(87.5)$ & $\mathrm{p}<0.001$ \\
\hline
\end{tabular}


Table 4. Comparison of SSc-ILD [SSc (+)] group with other CTD-ILD [SSc (-)] group

\begin{tabular}{|c|c|c|c|}
\hline Characteristic & SSC (-) n (\%) & $S S c(+) n(\%)$ & Statistical significance \\
\hline \multicolumn{4}{|l|}{ Gender } \\
\hline Male & $8(88.8)$ & $1(11.1)$ & Chi $(1)=1.2457$ \\
\hline Female & $50(71.4)$ & $20(28.5)$ & $\mathrm{p}=0.432$ \\
\hline \multicolumn{4}{|l|}{$H R C T$} \\
\hline NSIP & $35(68.6)$ & $16(31.3)$ & Chi $(1)=2.4480$ \\
\hline UIP & $19(76.0)$ & $6(24)$ & $\mathrm{p}=0.784$ \\
\hline HP & $3(100)$ & 0 & \\
\hline $\mathrm{OP}$ & $1(100)$ & 0 & \\
\hline CPEF & 1 & 0 & \\
\hline \multicolumn{4}{|c|}{ Rheumatoid factor } \\
\hline Absent & $31(60.7)$ & $20(39.2)$ & Chi $(1)=10.9691$ \\
\hline Present & $30(93.7)$ & $2(6.2)$ & $\mathrm{p}=0.001$ \\
\hline \multicolumn{4}{|l|}{ ANA } \\
\hline Absent & $53(81.5)$ & $12(10.4)$ & Chi $(1)=0.9568$ \\
\hline Present & $8(44.4)$ & $10(55.5)$ & $\mathrm{p}=0.005$ \\
\hline \multicolumn{4}{|l|}{ Ex SOB } \\
\hline Absent & $22(78.5)$ & $6(21.4)$ & Chi $(1)=0.5592$ \\
\hline Present & 39 (70.9) & $16(29.0)$ & $\mathrm{p}=0.601$ \\
\hline \multicolumn{4}{|l|}{ Cough } \\
\hline Absent & $25(73.5)$ & $9(26.4)$ & Chi $(1)=<.001$ \\
\hline Present & $36(73.4)$ & $13(26.5)$ & $\mathrm{p}=1$ \\
\hline Fatigue & $48(72.7)$ & $18(27.2)$ & Chi $(1)=0.0972$ \\
\hline Absent & $13(76.4)$ & $4(23.5)$ & $\mathrm{p}=1$ \\
\hline \multicolumn{4}{|l|}{ Present } \\
\hline \multicolumn{4}{|c|}{ Bilateral fine end-inspiratory crepitation } \\
\hline Absent & $33(82.5)$ & $7(17.5)$ & Chi $(1)=3.2147$ \\
\hline Present & $28(65.1)$ & $15(34.8)$ & $\mathrm{p}=0.086$ \\
\hline
\end{tabular}

\section{Discussion}

CTD-ILDs were responsible for vast majority of secondary ILD in Sri Lanka [7]. This is the first study to describe the characteristics of CTD-ILD patients attending to lung specialized tertiary care centre in Sri Lanka.

The mean age of 55.6 years of our sample was similar to available literature $[8,10,11,12]$. Female preponderance was demonstrated as expected since the CTD was well known to occur in female gender $[7,8,11,12]$.

Evidence suggests that in some instances, ILD is the initial clinical manifestation of CTD [10,12]. There were two patients who initially presented with ILD in our sample as well. However, the date of the initial diagnoses was recorded only in 55 patients. RA was the most prevalent subtype of CTD-ILD followed by SSc. RA-ILD was recognized as the commonest type in many studies conducted globally $[12,13]$. A recent study conducted in Sri Lanka reported similar findings [7]. However, a descriptive study conducted in Portugal reported SSc as the commonest CTD-ILD subtype [8].

In consistent with the existing literature, our study reveals that the predominant pattern of HRCT in CTDILD group was NSIP followed by UIP [8,12]. As expected, shortness of breath and cough were the commonest presenting symptoms found in patients [11]. At the same time, it is important to note significant proportion did not have expected symptoms, which signifies the importance of screening with LFT and HRCT even in asymptomatic group.

Pulmonary function test which is widely used as a screening tool in detecting ILD, revealed predominant restrictive pattern as reported in literature [7,8]. 
RA-ILD is an important entity that carries higher mortality than RA patients without ILD [14]. RA-ILD patients, who comprised the majority of our sample, reported clear female preponderance. Despite female predominance in RA, the largest RA-ILD study conducted in the UK has revealed almost equal gender distribution [9]. On the other hand, there were some studies showing female preponderance as well $[14,15]$ emphasizing the importance of conducting local research to identify epidemiological variations.

Globally, many studies demonstrated UIP pattern as the predominant HRCT pattern in RA-ILD [2,9,14,16,17,18]. In contrast, the majority of our patients had NSIP pattern in HRCT followed by UIP in approximately one third of patients. Recent study conducted in Sri Lanka also reported NSIP pattern as the commonest HRCT pattern closely followed by UIP [7]. There were few cases of drug induced $\mathrm{HP}$ and organizing pneumonia. Though it is rare [19] we reported one case with CPFE Combined pulmonary fibrosis and emphysema as well.

RA-UIP pattern carries poorer prognosis and increases all-cause mortality in RA patients [9]. Since the NSIP pattern is the commonest type, our RA-ILD patients may have better overall survival in contrast to other countries, which we have not addressed in our study.

Our study demonstrated statistically significant difference in the presenting age of RA-ILD and other CTDILD. RA-ILD presented in late fifties compared to other CTD-ILDs, which presented in early fifties.

It is an already known fact that high levels of Rheumatoid Factor (RF) and titers of anti-CCP increase the risk of ILD development in RA [8,9,20,21]. Confirming this, the majority of our RA-ILD patients had positive RF and that association was statistically significant. We have not analyzed Anti-CCP as it was available only in few patients, since it is not freely available in the government sector.

SSc-ILD is the major cause of mortality in SSc [22,23]. The mean age of the SSc-ILD was significantly lower than that of other CTD-ILD groups. This showed the early presentation of SSc ILD compared to other group. Female preponderance was clearly observed in our sample similar to other studies $[2,14,24]$. The majority of SSc-ILD group was symptomatic at the time of presentation. Similar to global evidence NSIP was the predominant HRCT pattern $[2,14,24]$.

\section{Limitations}

The present study was a single-center retrospective study; hence, generalizing the findings of the study needs to be done cautiously. Since evidence suggest that there are substantial differences in data obtained from prospective disease registries compared to single-center retrospective studies [12], we believe it is important to establish prospective disease registries and conduct future studies to explore the potential associations further. Since this study involved retrospective data, some of the relevant data could not be retrieved due to misplacement of reports and records as well as deficiencies in record keeping.

\section{Conclusions}

To the best of our knowledge this is the first study to describe CTD-ILD patients in Sri Lanka. Comparison of the present study findings with evidence from other countries revealed both similarities and differences. RAILD was the most frequent type of CTD-ILD observed in our center followed by SSc. The most striking finding is that the predominant NSIP pattern and clear female preponderance in RA-ILD compared to global data.

We recommend prospective multicenter studies to be carried out and prospective disease registries to be established to explore the epidemiological, clinical, radiological and prognostic characteristics of CTD-ILD in Sri Lanka.

\section{Abbreviations}

Connective Tissue Diseases associated ILD (CTD-ILD)

Rheumatoid arthritis (RA)

Systemic sclerosis (SSc)

Inflammatory myositis (IM)

Primary Sjögren's syndrome (pSS)

Mixed connective tissue disease (MCTD)

Interstitial Pneumonitis with Autoimmune Features (IPAF)

Nonspecific pneumonia (NSIP)

Usual interstitial pneumonia (UIP)

Organizing pneumonia (OP)

Diffuse alveolar damage (DAD)

Lymphocytic interstitial pneumonia (LIP)

High Resolution CT scan (HRCT)

\section{Declarations}

Ethics approval and consent to participate: Ethicalapproval was obtained from the Ethics Review Committee of the Medical Research Institute (MRI) in Sri Lanka. Informed written consent was taken from the participants prior to entering in the database.

Consent for publication: Not applicable.

Availability of data and materials: The datasets generated and analysed during the current study are available from the corresponding author on reasonable request.

Conflicts of interest: All authors declare that there are no conflicts of interest.

Funding: None. 


\section{References}

1. Flaherty KR, Khanna D. Idiopathic or connective tissue disease-associated interstitial lung disease: a case of HRCT mimicry. Thorax 2014; 69(3): 205-6.

2. Geerts S, Wuyts W, Langhe E, Lenaerts J, Yserbyt J. Connective tissue disease associated interstitial pneumonia: a challenge for both rheumatologists and pulmonologists. Sarcoidosis Vasc Diffuse Lung Dis. 2017; 34(4): 326-35.

3. J, Corte TJ, du Bois RM, Lee JS, et al. "ERS/ATS Task Force on Undifferentiated Forms of CTD-ILD”. An official European Respiratory Society/American Thoracic Society research statement: interstitial pneumonia with autoimmune features. Eur Respir J. 2015; 46(4): 976-87.

4. Tanaka N, Newell JD, Brown KK, Cool CD, Lynch DA. Collagen vascular disease-related lung disease: highresolution computed tomography findings based on the pathologic classification. J Comput Assist Tomogr. 2004; 28(3): 351-60.

5. Monaghan H, Wells AU, Colby TV, du Bois RM, Hansell DM, Nicholson AG. Prognostic implications of histologic patterns in multiple surgical lung biopsies from patients with idiopathic interstitial pneumonias. Chest 2004; 125(2): 522-6.

6. Grubben MJ, Kerstens PJ, Wiersma JM, Boerbooms AM, Festen J. Pleuro-pulmonary involvement in patients with connective tissue disease. The role of open lung biopsy. Neth J Med. 1993; 43(5-6): 269-76.

7. Madegedara D, Rathnayake S, Bandara A. Analysis of clinical profile, aetiology, classification and outcome of interstitial lung diseases at a single center of Sri Lanka - a descriptive study. International Journal of Research GRANTHAALAYAH 2020; 8(10): 1-14.

8. Fischer A, West SG, Swigris JJ, Brown KK, du Bois RM. Connective tissue disease-associated interstitial lung disease: a call for clarification. Chest 2010; 138(2): 251-6.

9. Kelly CA, Saravanan V, Nisar M, et al. British Rheumatoid Interstitial Lung (BRILL) Network. Rheumatoid arthritisrelated interstitial lung disease: associations, prognostic factors and physiological and radiological characteristics-a large multicentre UK study. Rheumatology (Oxford). 2014; 53(9): 1676-82.

10. u Y, Wang LS, Wei YR, et al. Clinical Characteristics of Connective Tissue Disease-Associated Interstitial Lung Disease in 1,044 Chinese Patients. Chest 2016; 149(1): 201-8.

11. Alhamad EH. Interstitial lung diseases in Saudi Arabia: A single-center study. Ann Thorac Med. 2013; 8(1): 33-7.
12. Singh S, Collins BF, Sharma BB, et al. Interstitial Lung Disease in India. Results of a Prospective Registry. Am J Respir Crit Care Med. 2017; 195(6): 801-13.

13. Fischer A, Brown K. Pulmonary manifestations of rheumatic disease: A comprehensive guide. Evaluation of lung disease in patients with connective tissue disease. Springer Science 2014: 13-23.

14. Bryson T, Sundaram B, Khanna D, Kazerooni EA. Connective tissue disease-associated interstitial pneumonia and idiopathic interstitial pneumonia: similarity and difference. Semin Ultrasound CT MR 2014; 35(1): 29-38.

15. Zhang Y, Li H, Wu N, Dong X, Zheng Y. Retrospective study of the clinical characteristics and risk factors of rheumatoid arthritis-associated interstitial lung disease. Clin Rheumatol. 2017; 36(4): 817-23.

16. Kim EJ, Collard HR, King TE Jr. Rheumatoid arthritisassociated interstitial lung disease: the relevance of histopathologic and radiographic pattern. Chest 2009; 136(5): 1397-1405.

17. Tansey D, Wells AU, Colby TV, et al. Variations in histological patterns of interstitial pneumonia between connective tissue disorders and their relationship to prognosis. Histopathology 2004; 44(6): 585-96.

18. Lee HK, Kim DS, Yoo B, et al. Histopathologic pattern and clinical features of rheumatoid arthritis-associated interstitial lung disease. Chest. 2005; 127(6): 2019-27.

19. Shaw M, Collins BF, Ho LA, Raghu G. Rheumatoid arthritisassociated lung disease. Eur Respir Rev. 2015; 24(135): 1-16.

20. Turesson C, Jacobsson LT, Sturfelt G, Matteson EL, Mathsson L, Rönnelid J. Rheumatoid factor and antibodies to cyclic citrullinated peptides are associated with severe extra-articular manifestations in rheumatoid arthritis. Ann Rheum Dis. 2007; 66(1): 59-64.

21. O’Dwyer DN, Armstrong ME, Cooke G, Dodd JD, Veale DJ, Donnelly SC. Rheumatoid Arthritis (RA) associated interstitial lung disease (ILD). Eur J Intern Med. 2013; 24(7): 597-603.

22. Cappelli S, Bellando Randone S, Camiciottoli G, De Paulis A, Guiducci S, Matucci-Cerinic M. Interstitial lung disease in systemic sclerosis: where do we stand? Eur Respir Rev. 2015; 24(137): 411-9.

23. Steen VD, Medsger TA. Changes in causes of death in systemic sclerosis, 1972-2002. Ann Rheum Dis. 2007; 66(7): 940-4.

24. Bussone G, Mouthon L. Interstitial lung disease in systemic sclerosis. Autoimmun Rev. 2011; 10(5): 248-55. 